Rodriguez, N., Safont-Mottay, C., \& Prêteur, Y. (2017). L'expression de soi en ligne à l'adolescence : socialisation entre pairs et quête identitaire. Bulletin de psychologie, 70 (5), 355368

\title{
L'expression de soi en ligne à l'adolescence : socialisation entre pairs et quête identitaire
}

\author{
Rodriguez Nancy ${ }^{\mathrm{a}}$ \\ Safont-Mottay Claire ${ }^{\text {a }}$
}

Prêteur Yves ${ }^{\text {a }}$

a Laboratoire de psychologie de la socialisation. Développement et travail, EA1697, équipe «Psychologie du
développement et de l'éducation familiale et scolaire », Université Toulouse 2 - Jean Jaurès, France.

\section{$\underline{\text { http://www.bulletindepsychologie.net }}$}

Résumé : L'expression de soi en ligne est une pratique très prisée des jeunes adolescentes, mais souvent questionnée pour ses effets sur le développement de l'individu. Dans une perspective compréhensive, cette recherche vise à appréhender en quoi ces pratiques d'expression de soi en ligne s'inscrivent dans un processus de socialisation horizontale et participent au développement identitaire. Des entretiens semi-directifs ont été menés auprès de 47 adolescentes âgées de 11 à 15 ans. Une analyse lexicométrique avec une classification hiérarchique descendante (logiciel Alceste) montre que cette pratique s'inscrit bien au cœur de la socialisation horizontale et est bien plus qu'un moyen d'intégration sociale. L'expression de soi en ligne, d'abord apprise auprès des pairs, est une pratique qui (se) personnalise. Elle permet la mobilisation de capacités réflexives sur soi, dans un travail intersubjectif, et reflète un réel désir de reconnaissance et de soutien social.

\section{Self-expression online in adolescence: Peers socialization and Identity quest}

Abstract: The self-expression online is a popular practice appreciated by teenage girls, but often questioned for its effects on the development of the individual. In a comprehensive approach, this research aims to understand how these practices of self-expression online fit into a horizontal socialization process and contribute to identity development. Semi-structured interviews were conducted with 47 teenage girls aged 11 to 15 years. A lexicometric analysis with a Hierarchical Descending Classification (Alceste software) shows that self-expression online fits well in the heart of the horizontal socialization and proves to be much more than a means of social integration. 
Self-expression online, first learned from peers, is a practice both personalizing and customizable. It allows the mobilization of self-reflexive abilities in an intersubjective work, and reflects a real desire of social recognition and support. 


\section{INTRODUCTION}

L'utilisation des nouvelles technologies de l'information et de la communication (NTIC) tient une place considérable, dans les pratiques de loisirs des adolescentes et des adolescents (Octobre, 2014). Avec l'avènement des ordinateurs portables, des tablettes tactiles et des smartphones, une nouvelle dynamique d'échange entre pairs s'est instaurée : il est possible de discuter avec des proches, quel que soit l'endroit et le moment, de renouer avec des amis d'enfance ou encore d'organiser des événements à distance (Lerolle, 2012 ; Moreau, Roustit, Chauchard, Chabrol, 2012). Le rapport à l'autre et au monde s'enracine désormais dans une culture de l'écran et du « tout et tout de suite» (Le Breton, 2011).

Facebook, Instagram, Skype et Snapchat sont devenus des outils relationnels (Cardon, 2011), des espaces de communication privilégiés par les jeunes (Balleys, 2015). Pour alimenter, entretenir les échanges entre pairs et attirer l'attention de l'autre, les adolescents se sont appropriés ces espaces dans une pratique d'expression de soi (Davis, 2012 ; Dolev-Cohen, Barak, 2013). En revisitant l'écriture, le dessin et la photographie, une véritable mise en scène de soi s'opère (Rodriguez, Safont-Mottay, Prêteur, 2013) : on se livre dans un récit du quotidien, on exprime ses émotions et ses états d'âme et on affirme ses liens amicaux auprès d'un réseau important de pairs.

Très médiatisée, cette pratique, répandue notamment à l'adolescence, est au centre d'un questionnement éthique, propre à la protection de la vie privée et à la diffusion d'informations personnelles sur Internet (Casilli, 2013). Nombre de chercheurs s'intéressent ainsi aux enjeux de l'expression de soi en ligne et examinent, par exemple, ses effets sur le bien-être du sujet, les représentations de soi ou encore l'acquisition de compétences sociales (Bach, Houdé, Léna, Tisseron, 2013 ; Israelashvili, Taejin, Buzoka, 2012 ; Lee, 2014 ; Pinar Tosun, 2012 ; Rui, Stefanone, 2013 ; Skues, Williams, Wise, 2012). Toutefois, ces recherches n'examinent pas ou 
peu le sens que les adolescents accordent à leurs pratiques en ligne. L'originalité de notre recherche réside dans une étude compréhensive de la pratique d'expression de soi sur Internet, en lien avec la construction identitaire et le processus de socialisation, dans le champ de la psychologie du développement.

\section{L’expression de soi en ligne : subjectivité et réflexivité identitaire du sujet}

À l'origine, l'expression de soi est un récit, un discours sur la façon dont le sujet se voit et sur ce qu'il ressent, pense ou fait (Lipiansky, 1998). Au sens large, tout message oral ou écrit, peut exprimer quelque chose sur celui qui l'émet : la place qu'il s'assigne, la relation au destinataire, son état émotionnel. L'expression de soi est chargée d'affectivité, faite de caractéristiques personnelles changeantes, attachantes, à rejeter ou à défendre : « mes amours, mes passions, mes goûts, mes intérêts, mes rejets, mes répulsions, mes haines, c'est moi » (Lipiansky, 1998, p. 126). Le récit de soi est une version subjective de la réalité de l'individu qui l'éprouve (Bruner, 2006). Sous différentes formes, ce récit devient une œuvre particulièrement complexe, qui élabore du sens sur l'expérience vécue au sein d'une culture (Bruner, 2002). Il relève d'un processus autobiographique par lequel l'individu reconceptualise, réorganise son passé et le fait coexister avec son avenir (Pineau, 2012). Par ce récit, l’individu est en mesure d'expliciter sa propre singularité et de prendre conscience de son existence (Bertaux, 1997 ; Delory-Momberger, 2009).

Pratiquée par des adolescents à l'aide de supports numériques, l'expression de soi en ligne prend ici une forme écrite et imagée. Son contenu renvoie à une multitude de traces de soi laissées spontanément sur la toile (Cardon, 2011). Cette pratique en ligne met notamment en lumière des conduites textuelles personnelles (style d'écriture, complexité des phrases, ton 
employé dans les messages...) adaptées selon les interlocuteurs (Guegan, Michinov, 2011 ; Walther, 2007). Ces conduites textuelles renvoient à la capacité d'adaptation du sujet selon le contexte dans lequel il se trouve et à la notion d'identité plurielle, puisqu'elles montrent différents sois possibles, exprimés ou exprimables à l'écrit (Walther, 2007).

L'expression de soi en ligne relève, à la fois, de l'ordre de l'invisible, par un travail de préparation, de modification ou de prise de distance par rapport aux publications, à l'abri des regards (Suler, 2004) et de l'ordre du visible, voire de l'extime (Tisseron, 2011) : le contenu à caractère intime, que le jeune publie sur lui-même, est rendu public et soumis volontairement à l'approbation des pairs. En acceptant de se livrer à l'autre, dans un cadre dyadique comme dans un cadre groupal ou même public (Davis, 2012), les adolescentes et les adolescents manifestent leurs désirs de soutien social (par commentaires et «likes ») et d'affirmation de soi (Pinar Tosun, 2012 ; Rui, Stefanone, 2013).

À l'adolescence en particulier, parce qu'ils sont perçus comme une source plus objective et relativement fiable d'informations, les pairs prennent une place importante dans la formation d'impressions, dans le développement des jugements et des représentations de l'individu sur lui-même et sur autrui (Deutsch, Gerard, 1955 ; Tafani, Falomir, Mugny, 2000). Les pairs détiennent alors un rôle clé : médiateurs dans la perception de la réalité, ils peuvent confirmer et consolider les représentations que l'individu se fait de lui-même, de ce qui l'entoure et des événements passés (McLean, Jennings, 2012). Pratiquer l'expression de soi en ligne permettrait ainsi, aux jeunes, d'appréhender de manière stratégique les perceptions de l'autre sur soi et d'anticiper des réactions négatives sur de futures publications (Dolev-Cohen, Barak, 2013 ; Lee, 2014 ; Pinar Tosun, 2012 ; Rui, Stefanone, 2013 ; Walther, 2007). Les adolescentes et les adolescents pourraient donc se faire une idée d'un « moi » acceptable. 
En ce sens plusieurs recherches en psychologie (Davis, 2012 ; Pinar Tosun, 2012 ; Wängqvist, Frisén, 2016) mettent en avant l'idée que l'expression de soi en ligne est une pratique qui accompagne la construction de l'identité à l'adolescence. Stimulée par le regard de l'autre et une forme de désirabilité sociale, cette pratique impliquerait une réflexion sur soi et sur la manière de se présenter à l'autre, des connaissances sur soi et une exploration des sois possibles (Skues et coll., 2012). Des supports d'expression de soi comme Facebook, semblent être devenus pour les adolescentes et les adolescents un moyen puissant d'impliquer l'autre dans son propre questionnement identitaire (« qui suis-je ? », « comment suis-je perçu ? », « quelle est ma place parmi les autres ?»).

\section{« Se faire soi » dans le rapport à l'autre et à l'intime}

L'adolescence est une période d'appropriation de nouveaux rôles sociaux, qui nécessite une prise de distance par rapport aux normes comportementales de l'enfance. Elle constitue un temps de socialisation majeur dans le développement de l'individu et concorde avec l'apparition de modifications des relations et des pratiques.

Conçue comme l'adaptation de l'individu aux institutions par sa participation progressive, dès l'enfance, à un jeu d'échanges complexes au sein des groupes auxquels il appartient (Malrieu, Malrieu, 1973), la socialisation est à la fois active, plurielle, conflictuelle et prospective. En effet, elle est une construction active du sujet par lui-même tout au long de sa vie, dans une pluralité de milieux sociaux (parfois source de contradictions et de conflits) et l'élaboration de projets de vie (Hugon, Villatte, Prêteur, 2013).

S'opérant de façon verticale (des parents vers l'enfant, du professeur vers l'élève), la socialisation, durant l'enfance et l'adolescence, repose également sur les interactions avec les 
pairs, sur un versant horizontal (Vasquez-Bronfman, Martinez, 1996). Le jeune s'ouvre au monde, s'intègre dans une pluralité de groupes de pairs et trouve de nouveaux repères, de nouvelles figures d'identification et d'attachement (Mallet, 2015). Il intériorise leurs normes, règles, valeurs, pratiques et exigences sociales et culturelles, tout en s'appropriant celles auxquelles il adhère le plus, en les personnalisant et en leur donnant du sens (Malrieu, BaubionBroye, Hajjar, 1991 ; Malrieu, Malrieu, 1973). De ce fait, Malrieu et Malrieu (1973) ou encore Tap (1991) évoquent une dynamique d'acculturation (intégration sociale et intériorisation des données sociales du groupe) et de personnalisation (appropriation et subjectivation des données sociales du groupe), dont la finalité est de trouver sa place, de se faire soi parmi les autres (Guichard, 2004). Cette dynamique d'acculturation et de personnalisation fait écho aux processus d'exploration et d'engagement identitaire (Marcia, 1966, 1993). L'adolescent expérimente la richesse sociale des groupes de pairs, leurs normes, leurs valeurs et leurs règles. Il s'engagerait ensuite sur celles qui le représentent le plus et auxquelles il accorde une réelle importance. L'interaction de ces deux processus forme l'identité du sujet dans une culture donnée (Kunnen, Bosma, 2006).

C'est plus particulièrement auprès des amis proches, dans un climat sécurisant et de confiance, que les adolescents testeront et affirmeront leurs idées, leurs goûts, leur style vestimentaire et leur image (Coslin, 2010 ; Mallet, 2015). L'écoute, l'ouverture de soi à l'autre, les confidences et la verbalisation des sentiments entre amis participent au développement d'une intimité relationnelle propice à l'exploration de nouvelles situations sociales et émotionnelles (Mallet, 2015). Les informations personnelles et intimes recueillies sur 1'autre, lors d'échanges entre amis et durant le temps passé ensemble, permettraient à l'adolescent de se comparer à l'autre, de faire état de leurs ressemblances et de leurs différences (Festinger, 1954 ; Guichard, 2004 ; Zimmermann, Quartier, 2013). Ce dialogue entre proches s'apparente 
à un travail intersubjectif et fait référence à la capacité du sujet à donner un sens à sa vie, à coconstruire avec l'autre le sens de la réalité en unifiant leurs perceptions du monde (Golse, 2014 ; Zimmermann, Quartier, 2013). L'intersubjectivité alimenterait la réflexion sur soimême, amenant les sujets à (re)signifier ensemble leurs conduites et les événements de leur vie. C'est en ce sens qu'elle est qualifiée d' « intersubjectivité réflexive » (Martin, Sokol, Elfers, 2008). Les ami(e)s intimes, deviennent ainsi, à l'adolescence, le miroir privilégié dans lequel le « moi » peut saisir certains de ses reflets, qu'ils soient physiques, émotionnels ou sociaux (Rodriguez-Tomé, 1972).

\section{OBJECTIFS}

Si la construction identitaire est une thématique souvent associée à l'expression de soi en ligne (Israelashvili et coll., 2012 ; Pinar Tosun, 2012 ; Rui, Stefanone, 2013 ; Wängqvist, Frisén, 2016), la socialisation par les pairs l'est encore peu. Notre étude, inscrite dans une démarche compréhensive, vise à examiner la manière dont ces thématiques de recherche, qui semblent étroitement liées, interagissent. Le regard subjectif de jeunes adolescents tout venant, pratiquant l'expression de soi, peut donc nous permettre d'interpréter et de décrire le sens de ces relations.

Les pratiques d'expression de soi en ligne se diversifiant selon le genre (Davis, 2012 ; Jousselme, Cosquer, Hassler, 2013 ; Metton-Gayon, 2009 ; Subrahmanyam, Greenfield, 2013), nous centrons cette recherche sur le vécu et l'expérience d'adolescentes, à un âge (11-15 ans) où elles possèdent leurs écrans personnels et découvrent de nouvelles façons d'interagir avec leurs pairs (Bigot, Croutte, Daudey, 2013 ; Lerolle, 2012). Ces dernières utilisent les réseaux sociaux et les messageries instantanées dans une pratique de publication personnelle et d'échanges intimes, de manière plus importante que les garçons, qui font davantage usage de 
ces outils pour partager de l'information et jouer en ligne avec leurs amis (Metton-Gayon, 2009).

En explorant la réalité subjective de jeunes adolescentes, dans une ouverture empathique, nous souhaitons observer comment leurs pratiques d'expression de soi en ligne, par les réseaux sociaux et les messageries instantanées, s'inscrivent ou s'articulent avec la socialisation horizontale de l'adolescente et soutiennent le développement identitaire. En cela, il s'agit d'examiner leurs représentations de leurs pratiques d'expression de soi en ligne, de l'amitié et de leur vécu amical, et leurs perceptions d'elles-mêmes. Nos hypothèses sont les suivantes :

- les pratiques d'expression de soi en ligne des adolescentes sont au cœur de la socialisation horizontale. En effet, les pratiques d'expression de soi sont, d'une part, vecteur de socialisation, en permettant aux adolescentes d'entretenir leurs relations amicales, de rester connectées en permanence à l'autre et, d'autre part, une pratique socialisante visant l'intégration sociale des jeunes et le renforcement de leur sentiment d'appartenance à des groupes (usage des mêmes outils entre amis) ;

- les expériences socialisantes vécues via les pratiques d'expression de soi en ligne, synonymes d'un travail intersubjectif, contribuent à accompagner le développement des représentations de soi qui participent à la construction identitaire ;

- les pratiques d'expression de soi en ligne, en tant que pratiques relationnelles, s'inscrivant dans une socialisation horizontale, constituent, pour les adolescentes, un support de coconstruction du sens de la réalité avec l'autre, de réflexivité intersubjective et, donc, une aide supplémentaire pour approfondir leurs représentations de l'amitié et d'elles-mêmes.

\section{MÉTHODOLOGIE DE LA RECHERCHE}




\section{Population}

Cinquante-quatre adolescentes, âgées d'11 à 15 ans, ont été sollicitées hors du contexte scolaire avec l'aide de deux associations sportives et de loisirs du Sud-Ouest de la France. Parmi ces adolescentes, 47 ont accepté de participer à cette recherche $(M=12,9 ; E T=1,141)$. Les participantes sont scolarisées au collège, de la classe de la sixième à la troisième $\left(6^{\mathrm{e}}=13 ; 5^{\mathrm{e}}=\right.$ $\left.9 ; 4^{\mathrm{e}}=11 ; 3^{\mathrm{e}}=14\right)$ et issues de milieux variés. Toutes sont utilisatrices régulières d'Internet, notamment des réseaux sociaux et des messageries instantanées.

\section{Procédure et outils}

Les adolescentes ont été rencontrées au printemps 2012, en présence de leur(s) parent(s), dans le cadre de journées d'ateliers organisées par les associations sportives et de loisirs. Après avoir obtenu l'accord de la participation des adolescentes et l'autorisation de leurs parents, un entretien semi-directif individuel a ensuite été programmé à leur domicile, la semaine suivante. Cet entretien individuel, d'une durée moyenne de 30 minutes, s'est déroulé dans une pièce calme du domicile, à l'abri des regards. La grille d'entretien a été orientée sur trois thématiques :

- l'utilisation d'Internet de chaque adolescente et leurs pratiques d'expression de soi en ligne (exemples : «La première fois que tu t'es connectée sur Internet, c'était pour quoi, tu t'en souviens ? », « Quel genre de choses t'arrive-t-il de publier en ligne ?»);

- leurs représentations de l'amitié et leur vécu amical quotidien (exemples : «Pour toi, qu'estce que c'est l'amitié ? Qu'est-ce que ça signifie ? », « Est-ce que tu peux m'en dire davantage sur ce que vous faites ensemble, si vous vous aidez, si vous vous soutenez, si vous vous défendez etc. », «Est-ce que tes relations amicales ont toujours été comme ça ? »); 
- leurs propres représentations d'elles-mêmes (exemples : « Est-ce que tu te confies souvent à quelqu'un en particulier? Qu'est-ce que tu dis de toi généralement à cette ou ces personnes ?», «Comment aimerais-tu être vue par les personnes qui t'entourent? ?).

\section{Stratégie d'analyse}

L'ensemble des entretiens a fait l'objet d'une analyse lexicométrique par le logiciel Alceste $^{2}$. Le logiciel Alceste est centré sur les ressemblances et dissemblances du vocabulaire et permet de dégager les thématiques les plus récurrentes d'un texte et d'observer comment celles-ci s'organisent entre-elles (Reinert, 1990). Le caractère inductif de cette technique nous a paru adapté à notre démarche compréhensive. Le logiciel découpe l'ensemble du corpus en unités de contexte élémentaire (UCE) composées d'une ou plusieurs lignes de texte, puis il repère les « mots pleins » (noms, adjectifs, verbes et adverbes) de chaque UCE, les réduit à leur racine et calcule ensuite leur fréquence d'apparition. Par une classification hiérarchique descendante (CHD), le logiciel construit des classes d'UCE, regroupées par la cooccurrence des mots qui les constituent. Ces classes représentent des « profils-types » de réponses (AubertLotarski, Capdevielle-Mougnibas, 2002), qu'il est possible de décrire, en fonction de leur vocabulaire spécifique et de la sélection des UCE les plus représentatives avec leur coefficient d'association respectif $\left(\chi^{2}\right.$ significatif au seuil de $\left.p<0,05\right)$. Après avoir ainsi catégorisé les réponses des sujets en fonction de la ressemblance de leur vocabulaire grâce à la CHD, le logiciel permet, dans un deuxième temps, d'établir une correspondance avec des variables extratextuelles, dites illustratives, comme les caractéristiques socio-biographiques des personnes interviewées.

\footnotetext{
2. Alceste : analyse des lexèmes cooccurents dans les énoncés simples d'un texte, version 4.7
} 
Les variables illustratives retenues pour notre étude sont : l'âge, l'outil préférentiel pour pratiquer l'expression de soi en ligne, la forme de la pratique, ainsi que le mode relationnel (ou la constitution du réseau de pairs) en ligne, afin de pouvoir mettre en évidence d'éventuelles différences dans les représentations et usages. Ces variables ont été dichotomisées pour faciliter le traitement du corpus (tableau 1), dans une étape préliminaire d'analyse (Rodriguez, 2014).

\section{(Tableau 1)}

Deux groupes d'âges sont distingués, le premier représentant l'entrée dans l'adolescence et le deuxième l'adolescence proprement dite. Les outils préférentiels d'expression de soi en ligne, cités par les adolescentes, sont le réseau social Facebook et la messagerie instantanée Skype (anciennement $M S N$ ). Chacune déclare utiliser préférentiellement l'un de ces deux outils, bien que les deux outils soient parfois utilisés simultanément $(78,7 \%$ des adolescentes interrogées font usage de Facebook et 80,8\% se connectent sur $M S N$ ). La pratique d'expression de soi peut prendre deux formes : une forme réservée ou peu fréquente (hebdomadaire) et une forme expansive, très régulière (jusqu'à plusieurs fois par jours). Enfin, deux modes relationnels ont été constitués en tenant compte du nombre moyen de contacts ajoutés sur l'outil préféré d'expression de soi en ligne : un premier mode est relativement restreint aux cercles d'amis proches, aux camarades, ainsi qu'à certains membres de la famille avec un nombre de contacts inférieur à 100 ; le second est plus large, avec un nombre de contacts supérieur à 100 et allant parfois jusqu'à un nombre extrême (jusqu'à 700 « amis » sur Facebook).

Les entretiens semi-directifs forment un corpus analysable d'environ 75 pages (plus de 273900 signes, espaces compris), sans les questions.

\section{RÉSULTATS}


Au sein du corpus d'entretiens, le logiciel a dénombré 1165 UCE dont 827 ont pu être réparties en 6 classes distinctes à l'issue de la CHD, soit 70,99\% de notre corpus. Le pourcentage des UCE retenues est légèrement supérieur à 70\%, seuil à partir duquel l'analyse est statistiquement significative (Reinert, 1990). Sous ce logiciel, la CHD crée des diagrammes, appelés dendrogrammes, utilisés pour illustrer l'articulation ou l'association de classes d'UCE similaires, générés par un regroupement hiérarchique. Le dendrogramme de notre corpus (figure 1) montre les oppositions et associations des classes entre-elles.

\section{(Figure 1)}

Le dendrogramme indique la présence de deux éclatements ou ramifications, qui opposent des groupes de classes entre elles. La première ramification distingue deux regroupements de classes. Un premier regroupement (classes 1 et 6) se rapporte à la thématique de l'amitié, au « faire ensemble » et au dialogue entre ami(e)s). Un deuxième regroupement (classes 2 et 3) porte sur les pratiques d'Internet et l'expression de soi en ligne. La seconde ramification est composée du troisième regroupement (classes 4 et 5), qui met en lien le regard que les adolescentes portent sur elles-mêmes et leurs représentations du rôle de l'autre dans la relation amicale. Afin d'en extraire les thèmes ou la dynamique sous-jacente, nous analyserons les formes les plus représentatives (vocabulaire et UCE caractéristiques) de chaque classe. Nous examinerons la présence des variables illustratives associées. Enfin, nous décrirons l'articulation des classes entre elles pour tenter de mieux comprendre leurs associations et leurs oppositions.

\section{Regroupement des classes 1 et 6}

Classe 1. Faire ensemble 
Les activités partagées entre amies, « le faire ensemble » constituent la première classe distinguée par l'analyse lexicométrique $(14,39 \%$ du corpus). Les participantes, nous renseignent sur le type d'activités et les moments privilégiés qu'elles consacrent à leurs ami(e)s. Les formes les plus représentatives de cette classe sont les suivantes : « journée » $\left(\chi^{2}=89,38\right.$, $\mathrm{p}<0,05)$, « vacances » $\left(\chi^{2}=71,62, \mathrm{p}<0,05\right)$, « soir » $\left(\chi^{2}=71, \mathrm{p}<0,05\right)$, « ensemble » $\left(\chi^{2}=70,1\right.$, $\mathrm{p}<0,05)$, « ciné » $\left(\chi^{2}=66,33, \mathrm{p}<0,05\right)$, « soutenir » $\left(\chi^{2}=56,91, \mathrm{p}<0,05\right)$, « défendre » $\left(\chi^{2}=52,99\right.$, $\mathrm{p}<0,05)$, « manger » $\left(\chi^{2}=46,98, \mathrm{p}<0,05\right)$, «dormir $»\left(\chi^{2}=46,98, \mathrm{p}<0,05\right)$, « rigoler $»\left(\chi^{2}=44,50\right.$, $\mathrm{p}<0,05)$, «week-end » $\left(\chi^{2}=35,37, \mathrm{p}<0,05\right)$ et « potin » $\left(\chi^{2}=35,05, \mathrm{p}<0,05\right)$.

L'ensemble de ces formes se rapporte aux relations amicales proches, au sein desquelles il est question d'entraide et de partage. Plusieurs repères temporels, notamment des repères journaliers, sont représentés et semblent propices aux confidences, au soutien moral et à un renforcement des liens amicaux : «On se soutient quand l'une de nous va pas bien, on essaye de la faire rire, des fois on part dans des délires tout bêtes mais ça change vite les idées » (A., 12 ans) ; « On se défend, on se soutient, on sort au ciné à côté de chez nous et on va à la piscine ensemble, on délire beaucoup surtout en sortant du ciné quand le film nous a plu » (E., 12 ans) ; « Dès qu'on peut on se parle ou on s'appelle, ou on essaye de se voir pour se raconter des potins » (N., 15 ans) ; « Tous les soirs on se retrouve sur MSN, un peu, pas beaucoup et on parle de tout et de rien, on continue de délirer, on parle des mecs biens ou moches, des trucs qui se sont passés au collège » (N., 12 ans). Aucune variable illustrative n'est associée à cette classe.

\section{Classe 6. Le dialogue entre ami(e)s}

La classe 6 porte sur le dialogue, les échanges quotidiens entretenus entre ami(e)s proches et les formes que prennent ces échanges (19,83\% du corpus). Cette classe se centre davantage que la première sur la qualité de la relation que les jeunes adolescentes entretiennent avec leur(s) meilleur(e-s) ami(e-s), l'histoire de la relation, leur complémentarité et le contenu intime des 
discussions qu'elles déclarent avoir régulièrement avec eux (elles). Voici les formes représentatives de cette classe : «meilleur » $\left(\chi^{2}=109,11, \mathrm{p}<0,05\right)$, « ami » $\left(\chi^{2}=92,04, \mathrm{p}<0,05\right)$, «parler » $\left(\chi^{2}=44,03, p<0,05\right)$, «nouveau » $\left(\chi^{2}=39,24, p<0,05\right)$, « collège $»\left(\chi^{2}=29,20, p<0,05\right)$, « face » $\left(\chi^{2}=28,13, \mathrm{p}<0,05\right)$, « classe $»\left(\chi^{2}=25,38, \mathrm{p}<0,05\right)$ « connaître $»\left(\chi^{2}=24,33, \mathrm{p}<0,05\right)$, « rencontre » $\left(\chi^{2}=23,85, \mathrm{p}<0,05\right)$, « téléphone » $\left(\chi^{2}=23,61, \mathrm{p}<0,05\right)$, « proche » $\left(\chi^{2}=21,08\right.$, $\mathrm{p}<0,05)$, « passion » $\left(\chi^{2}=20,27, \mathrm{p}<0,05\right)$, « primaire » $\left(\chi^{2}=17,70, \mathrm{p}<0,05\right)$, Internet $»\left(\chi^{2}=\right.$ $17,05, \mathrm{p}<0,05)$ et $«$ blues $»\left(\chi^{2}=16,25, \mathrm{p}<0,05\right)$.

Les adolescentes connaissent leurs amies parfois depuis de nombreuses années, celles-ci constituent un repère dans leur vie. Les adolescentes recherchent auprès de leur(s) ami(e-s) de la proximité et de l'intimité pour se confier. Leur entente est fondée sur les moments passés ensemble et la connaissance des multiples aspects de la vie de l'autre. Elles apprécient leurs échanges, les points communs qu'elles partagent, comme les différences qui les enrichissent. Elles restent également ouvertes aux nouvelles rencontres amicales : « À mes meilleures amies, je leur dis ce que je deviens et ce qui m'arrive de nouveau, et aux nouvelles [amies, rencontrées au collège] je leur raconte ce que j'ai vécu avant et, vu qu'on apprend à se connaître, on parle de nos passions » (I., 11 ans) ; « On a pas toutes les mêmes histoires et on a vécu des choses différentes et c'est bien d'avoir des amies différentes » (N., 12 ans) ; « J'ai des amies super mais c'est pas comme avec elle [meilleure amie], je la connais depuis longtemps. J'aime bien avoir des nouveaux amis, mais je veux la garder, surtout elle » (C., 12 ans) ; « Je dis à mes meilleures amies quand je vais pas bien, quand je suis malade, quand il s'est passé quelque chose » (M., 12 ans).

Dans cette classe, plusieurs variables illustratives sont représentées. Les extraits de discours les plus significatifs appartiennent à des jeunes adolescentes âgées de 11 et 12 ans $\left(\chi^{2}=3,99\right.$, 
$\mathrm{p}<0,05)$, utilisatrices des réseaux sociaux $\left(\chi^{2}=8,21, \mathrm{p}<0,05\right)$ dans une pratique d'expression de soi expansive $\left(\chi^{2}=2,74, \mathrm{p}<0,05\right)$.

La lecture des extraits du discours apporte un éclairage sur les associations soulevées avec ces variables. Les jeunes adolescentes âgées de 11 et 12 ans paraissent très actives dans l'exploration des liens sociaux et amicaux. La transition scolaire de l'école primaire au collège peut expliquer cette dynamique exploratoire. Les passions, l'intimité émotionnelle et sociale vécue font, par exemple, l'objet de discussions par lesquelles les adolescentes apprennent à connaître leurs nouvelles amies et maintiennent leurs amitiés de longue date. L'usage des réseaux sociaux, notamment dans le cadre d'une pratique d'expression de soi expansive, constitue un moyen, voire une stratégie relationnelle sur laquelle les adolescentes peuvent s'appuyer pour démarrer et poursuivre leurs conversations intimes entre amies.

\section{Regroupement des classes 2 et 3}

\section{Classe 2. Publications en ligne}

La classe 2 a trait à l'activité de publication des adolescentes en ligne (11,49\% du corpus). Les adolescentes décrivent leur pratique d'expression de soi. Elles expliquent ce qu'elles aiment publier, à quel rythme et avec quels outils d'Internet. Elles explicitent leur(s) motivation(s) et leurs attentes quant à la pratique de publications. Les formes représentatives de cette classe sont : « photos » $\left(\chi^{2}=266,94, \mathrm{p}<0,05\right)$, « mettre $»\left(\chi^{2}=128,30, \mathrm{p}<0,05\right)$, publier $»\left(\chi^{2}=108,42\right.$, $\mathrm{p}<0,05)$, « commentaire » $\left(\chi^{2}=102,26, \mathrm{p}<0,05\right)$, « écrire » $\left(\chi^{2}=73,99, \mathrm{p}<0,05\right)$, « page » $\left(\chi^{2}=\right.$ $54,40, \mathrm{p}<0,05)$, « journal » $\left(\chi^{2}=54,40, \mathrm{p}<0,05\right)$, « citation » $\left(\chi^{2}=54,40, \mathrm{p}<0,05\right)$, « commenter » $\left(\chi^{2}=54,23, \mathrm{p}<0,05\right)$, « textes » $\left(\chi^{2}=45,91, \mathrm{p}<0,05\right)$, " avis » $\left(\chi^{2}=38,76, \mathrm{p}<0,05\right)$ et « taguer » $\left(\chi^{2}=30,97, \mathrm{p}<0,05\right)$ 
Les extraits suivants illustrent la question du sens des pratiques, des motivations et des attentes d'adolescentes à propos de leurs écrits : « Je publie des paysages que je prends en photo parce que je vis à la campagne et qu'il y a des paysages super et souvent j'écris un petit texte assorti ou une citation de quelqu'un » (S., 13 ans) ; « Comme ça les autres ils savent un peu qui je suis et ce que j’ai vécu » (M., 11 ans) ; « Je les publie, comme ça je peux les faire voir à mes amis » (S., 14 ans) ; «J'attends d'avoir des commentaires, je reste pas longtemps connectée sur Facebook, mais je me connecte très souvent pour savoir si j'ai pas du nouveau sur mes publications » (E., 14 ans) ; « Des commentaires de temps en temps, mais j'en demande pas trop puisque je mets pas grand-chose [en ligne] » (N., 12 ans). L'expression de soi en ligne répond donc à des besoins particuliers. Pour certaines adolescentes, il s'agit de laisser une trace de leur vécu et de l'organiser, de donner des nouvelles aux personnes qu'elles côtoient plus rarement, de recueillir l'avis des pairs sur soi dans un souci de (re)connaissance.

Les extraits du corpus représentatifs de l'activité de publication en ligne et de la diversité des pratiques d'expression de soi viennent principalement d'adolescentes âgées de 13 à 15 ans $\left(\chi^{2}=2,07, p<0,05\right)$, utilisant les réseaux sociaux $\left(\chi^{2}=5,19, \mathrm{p}<0,05\right)$ et privilégiant un mode relationnel large $\left(\chi^{2}=3,93, \mathrm{p}<0,05\right)$.

Ces adolescentes ont plusieurs années d'expérience de l'utilisation d'Internet, notamment dans la pratique d'expression de soi. Dans une pratique régulière, elles ont eu la possibilité d'ouvrir et d'élargir leur réseau de pairs, en ajoutant, par exemple, plus de connaissances du milieu scolaire, leurs nouvelles amitiés nées d'un changement de classe, etc.

\section{Classe 3. L'expérience d'Internet}

La classe 3 aborde l'expérience d'Internet et l'évolution de la pratique chez les participantes (12,21\% du corpus). Les adolescentes présentent les outils et les sites Internet qu'elles ont 
l'habitude d'utiliser ( Facebook», «YouTube »). Elles évoquent leurs premières connexions et indiquent depuis combien de temps elles utilisent Internet. Voici les formes représentatives correspondantes : « an » $\left(\chi^{2}=267,92, \mathrm{p}<0,05\right)$, «Facebook» $\left(\chi^{2}=182,76, \mathrm{p}<0,05\right)$, « MSN» $\left(\chi^{2}=141,42, \mathrm{p}<0,05\right)$, « utiliser » $\left(\chi^{2}=133,42, \mathrm{p}<0,05\right)$, «vidéo » $\left(\chi^{2}=97,83, \mathrm{p}<0,05\right)$, «YouTube » $\left(\chi^{2}=93,77, \mathrm{p}<0,05\right)$, « site » $\left(\chi^{2}=71,70, \mathrm{p}<0,05\right)$, « recherches » $\left(\chi^{2}=65,40\right.$, $\mathrm{p}<0,05)$, « regarder » $\left(\chi^{2}=60,37, \mathrm{p}<0,05\right)$, « musique » $\left(\chi^{2}=57,31, \mathrm{p}<0,05\right)$, « aller $»\left(\chi^{2}=51,76\right.$, $\mathrm{p}<0,05)$, « souvenir » $\left(\chi^{2}=51,59, \mathrm{p}<0,05\right)$, ligne $»\left(\chi^{2}=50,89, \mathrm{p}<0,05\right)$, « droit $»\left(\chi^{2}=49,90\right.$, $\mathrm{p}<0,05)$ et $\ll \operatorname{servir} »\left(\chi^{2}=44,61, \mathrm{p}<0,05\right)$.

L'utilisation d'Internet, parfois récente ou plus ancienne, semble, quelquefois, coïncider avec une période de transition, un évènement important pour les adolescentes, par exemple, l'entrée au collège, une prise de responsabilité en début d'adolescence, une autorisation des parents : « Depuis plus de deux ans, en tout cas c'est depuis longtemps que je m'en sers mais je me souviens plus, c'était peut-être quand j'ai commencé le collège » (M., 14 ans) ; « J'ai le droit d'utiliser Internet depuis trois ans » (L., 14 ans) ; «Depuis tout juste un an, j'ai commencé l'été dernier» (I., 11 ans).

Les outils, que les adolescentes utilisent et qu'elles préfèrent pour s'exprimer et observer, sont multiples. Elles les manipulent, d'ailleurs, en parallèle : «J'adore aller sur YouTube, Facebook mais surtout Twitter » (M., 14 ans) ; « Je ne sais pas ce que je préfère le plus, je découvre plein de trucs qui sont bien » (H., 11 ans).

Les adolescentes décrivent leur initiation à leur outil préféré, dans laquelle les pairs et plus spécifiquement les meilleur(e)s ami(e)s sont impliquées : «Je m’en souviens pas, j’ai fait beaucoup de choses, je crois que je suis multitâches depuis toujours avec les ordis »(E., 12 ans) ; « [J'ai appris] par des amis. Ce sont eux qui m’ont expliqué comment ça marche et tout ça, en majeure partie, après je me suis débrouillée toute seule, j’ai appris un peu comme tout le 
monde » (T., 14 ans $)$; « [Des amis] m'en ont parlé plusieurs fois et à force de curiosité je suis allée voir et j'ai adopté » (F., 13 ans) ; " Avec mes copines, on s'est chacune fait un compte quasiment en même temps, on a appris ensemble » (L., 14 ans).

L'usage des réseaux sociaux et les messageries instantanées constituent des espaces sociaux à explorer, qui impliquent un apprentissage implicite de codes et de règles. L'utilisation de ces supports, dans une pratique spécifique d'expression de soi, est accompagnée par les pairs, qui motivent les adolescentes à consulter certains sites, qui les initient, leur transmettent ou construisent avec elles un ensemble de savoir-faire. Les adolescentes se guident et se conseillent entre elles. Aucune variable illustrative n'est associée à cette classe.

\section{Regroupement des classes 4 et 5}

\section{Classe 4. Le regard sur soi}

La classe 4 se centre sur le regard sur soi, regard que les adolescentes portent sur ellesmêmes en tenant compte du regard d'autrui. Cette classe est la plus importante puisqu'elle constitue $32,16 \%$ du corpus. Les adolescentes s'autoévaluent et expliquent en quoi elles se sentent bien dans leur peau. Elles décrivent les changements corporels, émotionnels et sociaux vécus, les transformations souhaitées sur leur apparence. Les adolescentes indiquent qu'elles tiennent compte à leur manière du regard des autres sur elles-mêmes, à travers les compliments et les critiques qui leurs sont adressés. De ce fait, certaines essayent ou veulent essayer de nouveaux styles vestimentaires. Les formes représentatives de cette classes sont : « critiquer » $\left(\chi^{2}=101,41, p<0,05\right), 《$ compliment $»\left(\chi^{2}=100,97, p<0,05\right)$, « changer $»\left(\chi^{2}=100,18, p<0,05\right)$, « fille » $\left(\chi^{2}=75,31, \mathrm{p}<0,05\right)$, « peau » $\left(\chi^{2}=54,40, \mathrm{p}<0,05\right)$, « timide » $\left(\chi^{2}=40,11, \mathrm{p}<0,05\right)$, « physique » $\left(\chi^{2}=40,11, \mathrm{p}<0,05\right)$, « normal » $\left(\chi^{2}=40,11, \mathrm{p}<0,05\right)$, « essayer » $\left(\chi^{2}=37,79\right.$, 
$\mathrm{p}<0,05)$, « gentille » $\left(\chi^{2}=36,61, \mathrm{p}<0,05\right)$, « sentir $»\left(\chi^{2}=35,58, \mathrm{p}<0,05\right)$, attentif $»\left(\chi^{2}=34,69\right.$, $\mathrm{p}<0,05)$, « tête » $\left(\chi^{2}=30,43, \mathrm{p}<0,05\right)$ et « jolie » $\left(\chi^{2}=30,03, \mathrm{p}<0,05\right)$.

Les extraits suivants illustrent des réflexions personnelles, des représentations d'adolescentes à propos d'elles-mêmes : « Je suis quelqu'un de bien et qui aime aider les autres et partager des moments simples avec eux »(E., 14 ans) ; «J'aimerais bien qu'on me voit comme une fille cool, ouverte, sympa, avec qui on peut parler, et qui est pas complexée. J'aimerais pas qu'on sente que j'ai des complexes parce que je sentirai que les autres voient que j'ai des failles ou des blessures » (A., 15 ans) ; « Je me suis toujours sentie comme ça, j’ai pas trop changée physiquement, juste pris des centimètres et puis j'ai eu des petites formes, je suis devenue une jeune fille» (L., 13 ans) ; «Je sais que les critiques c'est des fois pour nous booster donc j'y fais attention » (F., 12 ans). Le discours des participantes soulève un questionnement multidimensionnel sur le soi, particulièrement sur les aspects physiques, sociaux et psychologiques. Les adolescentes prennent conscience des transformations qu'elles vivent ou qu'elles ont vécues. Elles se caractérisent très souvent par les termes «cool », « ouverte », « sociable », « timide », « à l'écoute », etc. De manière implicite, les adolescentes se comparent et se décrivent par rapport à l'autre. De ce fait, la construction identitaire des adolescentes s'inscrit dans le rapport à l'autre : elles décrivent leur(s) rôle(s) et leur position parmi les autres. Aucune variable illustrative n'est associée à cette classe.

\section{Classe 5. Le rôle de l'autre}

Le rôle de l'autre, dans la relation amicale, constitue la classe 5 (9,92\%du corpus). Dans cette classe, les adolescentes expliquent leur conception de l'amitié et de l'ami(e), leurs attentes dans une relation amicale et les valeurs auxquelles elles sont attachées. Les mots récurrents de cette classe sont connotés à la confiance, à la compréhension, à la réciprocité, au partage, aux moments passés ensemble. Les formes représentatives de cette classe sont les suivantes : 
« amitié » $\left(\chi^{2}=217,69, \mathrm{p}<0,05\right)$, «bon » $\left(\chi^{2}=105,74, \mathrm{p}<0,05\right)$, « mauvais » $\left(\chi^{2}=94,87, \mathrm{p}<0,05\right)$, « confiance » $\left(\chi^{2}=65,70, p<0,05\right)$, « soutien » $\left(\chi^{2}=64,14, p<0,05\right)$, « vrai » $\left(\chi^{2}=51,83, p<0,05\right)$, « moments » $\left(\chi^{2}=51,83, \mathrm{p}<0,05\right)$, " précieux » $\left(\chi^{2}=45,70, \mathrm{p}<0,05\right)$, « partage » $\left(\chi^{2}=39,41\right.$, $\mathrm{p}<0,05)$, « réciproque » $\left(\chi^{2}=38,31, \mathrm{p}<0,05\right)$, « confier $»\left(\chi^{2}=36,87, \mathrm{p}<0,05\right)$, « solidarité » $\left(\chi^{2}=\right.$ $36,52, \mathrm{p}<0,05)$, , secret $»\left(\chi^{2}=28,43, \mathrm{p}<0,05\right)$ et $«$ besoin $»\left(\chi^{2}=27,58, \mathrm{p}<0,05\right)$.

Le discours tenu sur la représentation de l'amitié est similaire d'une jeune adolescente à l'autre. Elles s'appuient sur des valeurs universelles et décrivent quel est le rôle d'un(e) vrai(e) ami(e). En voici quelques exemples : «Une vraie amie c'est une confidente, c'est une personne qui est là dans les bons moments comme dans les mauvais, c'est comme un double à qui on peut tout dire sans avoir la peur d'être jugée ou trahie » (C., 12 ans) ; « [L'amitié] pour moi c'est quand on peut compter sur quelqu'un et quand c'est réciproque, c'est du soutien dans les bons et les mauvais moments, c'est des moments de secrets et de rigolade » (D., 14 ans). Certaines vont plus loin dans leur réflexion, et pensent que sans l'amitié, la vie n'aurait aucun sens : «L'amitié pour moi c'est quelque chose qui permet d'avancer, c'est beaucoup de confiance, c'est des moments à partager qu'ils soient bons ou mauvais. Moi je pense qu'on peut pas vivre sans amis et sans amitié, je sais pas mais je crois qu'on n'existerait pas sans ça » (S., 15 ans).

Les extraits de discours les plus significatifs de cette classe proviennent d'adolescentes âgées de 13 à $15\left(\chi^{2}=5,09, p<0,05\right)$, qui utilisent les réseaux sociaux $\left(\chi^{2}=10,08, p<0,05\right)$ dans un mode relationnel large $\left(\chi^{2}=4,02, \mathrm{p}<0,05\right)$.

Ces adolescentes, qui utilisent préférentiellement les réseaux sociaux, semblent bien faire la distinction entre les relations amicales proches et les autres formes de relations interpersonnelles (les camarades, les connaissances, etc.). Si leur réseau de pairs est large, la définition qu'elles donnent de l'amitié se centre sur des valeurs fondamentales et universelles. 
L'ensemble des contacts « collectionnés » sur un réseau n'est pas toujours représentatif de leur perception de l'amitié. En ce sens, les réseaux sociaux et les messageries instantanées ne sont pas uniquement des outils grâce auxquels les adolescentes peuvent entretenir des liens amicaux intimes. Il est possible que les adolescentes constituent leur réseau de pairs en fonction de ce que l'autre peut leur apporter (de l'assurance, un regard extérieur plus neutre...) en réponse à des besoins de reconnaissance et d'intégration sociale. Leur âge plus avancé peut aussi jouer un rôle dans leur perception de l'amitié : l'expérience de l'exploration des liens sociaux et amicaux pourrait justement permettre aux adolescentes de préciser et d'enrichir leurs représentations.

\section{Synthèse des résultats de l'analyse lexicométrique}

La première ramification du dendrogramme, qui regroupe les classes 1, 6, 2 et 3, montre comment la créativité en ligne et l'utilisation d'Internet s'inscrivent dans le processus de la socialisation horizontale, dans la mesure où ces pratiques ont été initiées par les pairs, constituent une activité relationnelle et amicale et des moments partagés entre amies. Les adolescentes attribuent une place importante aux ami(e)s, leur procurant un soutien considérable, une attention et une intimité recherchées, propices aux confidences. Dans ce cadre, les adolescentes sont encouragées à « faire ensemble », à poursuivre leurs récits et leurs tentatives de présentation de soi en ligne, à explorer la toile comme nouveau terrain d'interaction. La possibilité d'être en contact permanent avec des ami(e)s proches via des supports d'expression de soi en ligne est, pour les adolescentes, un atout pour obtenir du soutien social et une écoute attentive lorsqu'elles le souhaitent.

Les résultats de l'analyse lexicométrique indiquent donc une interaction entre les deux premiers regroupements (classes 1, 6, 2, 3), opposés à la seconde ramification du 
dendrogramme associant les classes 4 et 5 , le regard sur soi et le rôle de l'autre. Ce dernier regroupement indique que le regard que les adolescentes portent sur elles-mêmes est indissociable du regard de l'autre et du rôle qu'elles leur attribuent. Le rôle de l'autre est précieux dans la (re)connaissance des adolescentes sur elles-mêmes puisque qu'il coconstruit avec elles le sens de la réalité.

Par conséquent, les résultats suggèrent de différencier le processus de socialisation horizontale, dans lequel s'inscrivent les pratiques d'Internet (première ramification), et la construction de l'identité et des représentations de soi dans le rapport à l'autre (seconde ramification). Ces thématiques, mises en parallèle, interagissent, car les classes relatives à la « présence de l'autre » $(1,5$ et 6$)$ sont repérées sur les deux ramifications du dendrogramme.

\section{DISCUSSION}

Les principaux résultats inscrivent l'expression de soi en ligne dans le processus de la socialisation horizontale. De même, le regard porté sur soi et le rôle de l'autre sont associés, ce qui manifeste un mouvement intersubjectif dans la construction de l'identité.

\section{Les pratiques d'Internet au sein de la socialisation horizontale}

Les pratiques d'expression de soi s'ancrent dans une socialisation horizontale. Grâce à l'expression de soi et aux supports utilisés pour ces pratiques, les adolescentes s'intègrent dans des réseaux de pairs, intériorisent leurs normes, valeurs, pratiques et croyances tout en les personnalisant, en référence au modèle de la socialisation (Malrieu, Malrieu, 1973 ; Tap, 1991). Les adolescentes construisent leurs pratiques d'expression de soi ensemble, à l'aide de celles des ami(e)s et des aîné(e)s qui leurs servent de modèle. Ces pratiques se transmettent par les 
pairs, quelques fois par la fratrie, qui leur apprennent à maîtriser les outils privilégiés par les jeunes et l'activité de publication. Les adolescentes sont encouragées à « faire ensemble », en présence et par écrans interposés (Morgan, Korobov, 2012). Elles explorent la toile, parfois à plusieurs, comme un nouveau terrain social et expérimentent la publication de contenus personnels et intimes sous le regard d'autrui (Davis, 2012 ; Moreau et coll., 2012). L'activité de publication, qu'elle soit régulière ou rare, offre aux adolescentes l'occasion d'affirmer leurs goûts, tout en prenant plaisir à les partager (Israelashvili et coll., 2012 ; Pinar Tosun, 2012). Ces pratiques font donc l'objet d'un tâtonnement, d'un apprentissage informel de normes, de règles et de valeurs observées sur la toile. En ce sens, cette dimension d'apprentissages informels, de partage autour des pratiques, relie bien l'expression de soi en ligne à la socialisation entre pairs.

\section{Le regard de l'autre, ou l'intersubjectivité dans la construction identitaire}

La dimension intersubjective dans la construction identitaire (Golse, 2014 ; Martin, Sokol, Elfers, 2008 ; Zimmermann, Quartier, 2013) est clairement soulignée dans notre étude. Le regard que les adolescentes portent sur elles-mêmes est indissociable du regard de l'autre et du rôle qu'elles leur attribuent. Les adolescentes s'appuient sur les remarques des pairs et tiennent compte des avis extérieurs, plus particulièrement s'ils proviennent de pairs proches et valorisés, ces pairs qui constituent, pour elles, une source relativement fiable d'informations sur une situation donnée (Deutsch, Gerard, 1955 ; McLean, Jennings, 2012 ; Tafani et coll., 2000). Le groupe de pairs proche, au sein duquel des influences sociales normatives prennent souvent place (Deutsch, Gerard, 1955), peut, ici, servir de support de singularisation pour une adolescente en quête de son identité et peut l'encourager à exprimer ses opinions, ainsi que ses attentes envers les autres. En prenant connaissance du point de vue des pairs sur leur propre image, en s'adaptant à leurs attentes et aux pratiques valorisées, et en interagissant avec eux, 
les adolescentes maintiennent une dynamique de construction « moi - autre » (Guichard, 2004 ; Golse, 2014 ; Zimmermann, Quartier, 2013).

\section{Entre socialisation horizontale et construction identitaire dans l'intersubjectivité}

Notre recherche nous permet d'observer combien cette forme de socialisation horizontale, qui intègre les activités partagées entre pairs, comme les pratiques d'expression de soi en ligne, source d'une « intersubjectivité réflexive »(Martin, Sokol, Elfers, 2008 ; Zimmermann, Quartier, 2013), semble bien concourir à la construction identitaire à cette période du développement. Les résultats font allusion à une forme d'interstructuration du sujet et des contextes dans lesquels il interagit (Malrieu et coll., 1991). L'adolescente construit son identité, élabore ses représentations dans un contexte relationnel. La relation à l'autre est elle-même ancrée dans une socialisation horizontale, faite de pratiques, de valeurs, de normes et de croyances partagées entre pairs. En considérant Internet et l'outil numérique comme un contexte de vie, au sein duquel des interactions entre pairs ont lieu, les adolescentes s'adaptent et s'enrichissent des valeurs, normes et pratiques véhiculées par les pairs. En les adoptant, les adolescentes participent et interagissent avec le contexte et en suggèrent, à leur tour, de nouvelles. Au cœur des pratiques d'expression de soi, les adolescentes coconstruisent, avec les pairs, le sens de cette réalité, ainsi que leur rapport aux autres et au monde (Guichard, 2004 ; McLean, Jennings, 2012). L'expression de soi relève des activités créatives et d'invention que décrit Meyerson (1995) : l'expression de soi en ligne permet de laisser une trace de son passage, d'objectiver la réalité, sous une forme et un langage en harmonie avec celles de son époque.

\section{Une perspective développementale sous-jacente}


L'étude apporte également des éléments de réflexion quant à la question de l'âge. Les adolescentes âgées de 13 à 15 ans sont celles qui parlent le plus de la pratique de publication en ligne et du rôle de l'autre en tant qu'ami(e), tandis que les plus jeunes, âgées de 11 et 12 ans, sont celles qui représentent le mieux le dialogue entre ami(e)s. Nous pouvons en déduire que les plus jeunes se centrent davantage sur des pratiques qui leur permettent de rester en contact avec leurs proches, qui favorisent les échanges et les activités partagées entre ami(e)s. Ces jeunes adolescentes sont dans un état d'esprit de découverte, d'expérimentation de leur sociabilité en présence de proches qui les mettent en confiance et les rassurent sur leurs conduites, tout en s'initiant à des formes d'expression de soi émanant de plus âgées. Un processus d'exploration identitaire (Marcia, 1966 ; Kunnen, Bosma, 2006) semble être ici à l'œuvre. Les plus âgées, plus expérimentées dans la relation amicale et la gestion du regard de l'autre, paraissent pouvoir personnaliser leurs pratiques, en publiant, non plus pour faire avec et comme l'autre, mais pour elles-mêmes. Quelques manifestations d'engagement identitaire (Marcia, 1966 ; Kunnen, Bosma, 2006) sont ainsi repérables chez les plus âgées, notamment en ce qui concerne les valeurs de l'amitié et le rôle de l'autre dans la relation amicale.

\section{CONCLUSION}

Notre étude a exploré la manière dont les pratiques d'expressions en ligne s'inscrivent et prennent sens dans la socialisation horizontale. Sa réalisation a permis de mettre en lumière le rôle clé de cette forme de socialisation et l'intersubjectivité qu'elle sous-tend dans l'accompagnement et le développement de l'identité.

Bien plus qu'une sorte de stratégie pour attirer le regard de l'autre, l'expression de soi en ligne est une pratique adolescente permettant le dialogue et l'insertion des adolescentes au sein 
des groupes de pairs. Le temps passé ensemble en ligne permettrait d'entretenir le processus de comparaison sociale, une prise de position ainsi qu'une définition de soi parfois visible aux yeux de tous et soumise à l'approbation des pairs et ce de manière complémentaire aux situations hors-ligne.

Au terme de cette étude à visée compréhensive, plusieurs perspectives de recherche se dégagent. Tout d'abord, de nombreuses questions se posent quant à l'homogénéité des populations étudiées. En effet, selon le milieu et la culture d'appartenance, les normes, règles et valeurs des pratiques d'expression de soi en ligne, ainsi que les moyens d'accès à la pratique elle-même (matériel, condition d'accès à Internet...) peuvent différer (Lee, 2014).

Dans le cadre de cette recherche, les participantes sont de classes moyennes diversifiées. Elles avaient toutes un accès à Internet et la plupart possédaient déjà un écran personnel (smartphone, tablette, ordinateur) ou bien un matériel familial utilisable à l'abri des regards. Bien que les pratiques d'expression de soi en ligne soient riches et variées, celles-ci sont peutêtre représentatives d'une société occidentale « connectée ». En ce sens, les milieux de vie des adolescentes devraient faire l'objet d'une plus grande attention en tenant compte, par exemple, des formes que peuvent prendre ces pratiques et leurs liens avec l'identité et la socialisation horizontale selon la culture d'appartenance.

Les pratiques d'expression de soi sont très présentes chez les adolescentes, et elles existent aussi chez les garçons (Davis, 2012 ; Jousselme et coll., 2013 ; Metton-Gayon, 2009 ; Subrahmanyam, Greenfield, 2013). Ces pratiques d'expression de soi en ligne mériteraient d'être appréhendées chez des garçons du même âge et comparées à celles d'une population d'adolescentes, afin de voir si, chez des garçons, les pratiques d'expression de soi s'inscrivent de la même manière au cœur de la socialisation horizontale et jouent un rôle similaire dans la construction identitaire et la réflexivité sur soi. 
Une autre question, qui reste sans réponse, est de savoir comment les interactions sociales en ligne influencent la narration de soi. Proposer, aux adolescentes et aux adolescents, un temps de pratique en présence du chercheur, en individuel, permettraient de saisir la pluralité des stratégies mises en place par les jeunes dans leur activité d'expression de soi (adaptation du style d'écriture et travail sur les aspects imagés, photographiques des publications) et de voir comment ces jeunes réagissent face aux avis des pairs (filtrage, prise de distance). Des groupes d'adolescents et d'adolescentes pourraient être interrogés, animés par des psychologues pour examiner comment ces pratiques d'expression de soi en ligne se transmettent entre pairs proches dans le « faire ensemble » et circulent d'un groupe à l'autre.

\section{RÉFÉRENCES}

Aubert-Lotarski (Angeline), Capdevielle-Mougnibas (Valérie).- Dialogue méthodologique autour du logiciel Alceste en sciences humaines et sociales : "lisibilité » du corpus et interprétation des résultats, VIes Journées internationales d'analyse statistique de données textuelles, 2002 [le 22 février 2016, http://lexicometrica.univ-paris3.fr/jadt/jadt2002/PDF2002/aubert_capdevielle.pdf].

Bach (Jean-François), Houdé, (Olivier), Léna (Pierre), Tisseron (Serge).- L'enfant et les écrans. Avis de l'académie des sciences, Paris, Éducation Le Pommier, 2013.

Balleys (Claire).- Grandir entre adolescents à l'école et sur Internet, Lausanne, Presses polytechniques et universitaires romandes, 2015.

Bertaux (Daniel).- Les récits de vie, Paris, Armand Colin, 1997. 
Bigot (Régis), Croutte (Patricia), Daudey (Émilie).- La diffusion des technologies de l'information et de la communication dans la société française, CREDOC, Paris, Collection des rapports, 2013.

Bruner (Jérôme).- Pourquoi nous racontons-nous des histoires ?, Paris, Retz, 2002.

Bruner (Jérôme).- La culture, l'esprit et les récits, Enfance, 2, 2006, p. 118-125.

Cardon (Dominique).- Réseaux sociaux de l'Internet, Communications, 88, 1, 2011, p. 141148.

Casilli (Antonio A.).- Contre l'hypothèse de la « fin de la vie privée », Revue française des sciences de l'information et de la communication, 3, 2013.

Coslin (Pierre G.).-Psychologie de l'adolescent, Paris, Armand Colin, 2010.

Davis (Katie).- Friendship 2.0: Adolescents' experiences of belonging and self-disclosure online, Journal of Adolescence, 35, 6, 2012, p. 1527-1536.

Delory-Momberger (Christine).- La condition biographique. Essais sur le récit de soi dans la modernité avancée, Paris, Téraèdre, 2009.

Deutsch (Morton), Gerard (Harold B.).- A study of normative and informational social influences upon individual judgment, The Journal of Abnormal and Social Psychology, 51, 3, 1955, p. 629-636.

Dolev-Cohen (Michal), Barak (Azy).- Adolescents' use of Instant Messaging as a mean of emotional relief, Computers in Human Behavior, 29, 1, 2013, p. 58-63.

Festinger (Leon).- A theory of social comparison processes, Human Relations, 7, 1954, p. 117-140. 
Golse (Bernard).- De l'intersubjectivité à la subjectivation : un exemple de passage de l'interpersonnel à l'intrapsychique, Enfances \& Psy, 62, 1, 2014, p. 29-38.

Guegan (Jérôme), Michinov (Estelle).- Communication via Internet et dynamiques identitaires : une analyse psychosociale, Psychologie française, 56, 4, 2011, p. 223-238.

Guichard (Jean).- Se faire soi, L'orientation scolaire et professionnelle, 33, 4, 2004, p. 499533.

Hugon (Mandarine), Villatte (Aude), Prêteur (Yves).- Philippe Malrieu : un modèle de la socialisation-personnalisation, dans Baubion-Broye (A.), Dupuy (R.), Prêteur (Y.), Penser la socialisation en psychologie, Toulouse, Erès, 2013, p. 31-51.

Israelashvili (Moshe), Taejin (Kim), Buzoka (Gabriel).- Adolescents'over-use of the Cyberworld. Internet addiction or identity exploration? Journal of Adolescence, 35, 2012, p. $417-424$

Jousselme (Catherine), Cosquer (Mireille), Hassler (Christine).- Portraits d'adolescents. Enquête épidémiologique multicentrique en milieu scolaire en 2013, Paris, INSERM et Fondation Vallée Centre hospitalier, 2013.

Kunnen (Saskia E.), Bosma (Harke A.).- Le développement de l'identité : un processus relationnel et dynamique, L'orientation scolaire et professionnelle, 35, 2006, p. 183-203.

Le Breton (David).- Sur les cultures adolescentes, Le journal des psychologues, $\mathrm{n}^{\circ} 293,10$, 2011, p. 26-31.

Lee (Sangyup Y.).- How do people compare themselves with others on Social Network Sites? The case of Facebook, Computers in Human Behavior, 32, 2014, p. 253-260. 
Lerolle (Marie-Laure).- Junior Connect'. Tout savoir sur la fréquentation média et les comportements de consommation des jeunes de moins de 20 ans, Paris, IPSOS MediaCT, X 2012.

Lipiansky (Edmond-Marc).- Expression de soi, émotion et changement, dans Barbier (J. M.), Galatanu (O.), Action, affects et transformation de soi, Paris, Presses universitaires de France, 1998, p. 125-148.

Mallet (Pascal).- L'amitié entre enfants ou adolescents. Une force pour grandir, Paris, Armand Colin, 2015.

Malrieu (Philippe), Baubion-Broye (Alain), Hajjar (Violette).- Le rôle des œuvres dans la socialisation de l'enfant et de l'adolescent, dans Tap (P.), Malewska-Peyre (H.), La socialisation de l'enfance à l'adolescence, Paris, Presses universitaires de France, 1991, p. 163191.

Malrieu (Philippe), Malrieu (Suzanne).- La socialisation, dans Gratiot-Alphandery (H.), Zazzo (R.), Traité de psychologie de l'enfant, tome 5, Paris, Presses universitaires de France, 1973, p. 9-40.

Marcia (James).- Development and validation of ego identity status, Journal of Personality and Social Psychology, 3, 1966, p. 551-558.

Marcia (James).- The ego identity status approach to ego identity, dans Marcia (J.), Waterman (A. S.), Matteson (D. R.), Archer (S. L.), Orlovski (J. L.), Ego identity: A handbook for psychosocial research, New York, Springer, 1993, p. 1-21.

Martin (Jack), Sokol (Bryan W.), Elfers (Theo).- Taking and Coordinating Perspectives: From Prereflective Interactivity, through Reflective Intersubjectivity, to Metareflective Sociality, Human Development, 51, 2008, p. 294-317. 
McLean (Katie C.), Jennings (Lauren E.).- Teens telling tales: How maternal and peer audiences support narrative identity development, Journal of Adolescence, 35, 2012, p. 14551469.

Metton-Gayon (Céline).- Usages sexués d'Internet chez les adolescents et les modes de socialisation familiaux, Recherches \& éducations, 2, 2009, p. 163-177.

Meyerson (Ignace).- Les fonctions psychologique et les æuvres, Paris, Albin Michel, 1995.

Moreau (Agnès), Roustit (O.), Chauchard (Emeline), Chabrol (Henri).- L'usage de Facebook et les enjeux de l'adolescence : une étude qualitative, Neuropsychiatrie de l'enfance et de l'adolescence, 60, 6, 2012, p. 429-434.

Morgan (Elisabeth M.), Korobov (Neill).- Interpersonal Identity Formation in Conversations with Close Friends About Dating Relationships, Journal of Adolescence, $X X X V$, 6, 2012, p. 1471-1483.

Octobre (Sylvie).-Deux pouces et des neurones. Les cultures juvéniles de l'ère médiatique à l'ère numérique, Paris, Ministère de la Culture - DEPS, 2014.

Pinar Tosun (Leman).--Motives for Facebook use and expressing “True Self” on the Internet, Computers in Human Behavior, 28, 2012, p. 1510-1517.

Pineau (Gaston).- Histoire de vie et formation de soi au cours de l'existence, Société, 4, 118, 2012, p. 39-47.

Reinert (Max).- Alceste. Une méthodologie d'analyse des données textuelles et une application : Aurélia de Gérard de Nerval, Bulletin de méthodologie sociologique, 26, 1990, p. 24-54 
Rodriguez (Nancy).- Identité, représentations de soi et socialisation horizontale chez des adolescentes âgées de 11 à 15 ans pratiquant l'expression de soi sur Internet, thèse de doctorat nouveau régime en psychologie (non publiée), Université Toulouse 2 Jean Jaurès, Toulouse, 29 septembre 2014.

Rodriguez (Nancy), Safont-Mottay (Claire), Prêteur (Yves).- Tenir un blog à la préadolescence : enjeux identitaires dans une socialisation active et singulière, Recherches $\&$ éducations, 8, 2013, p. 149-164.

Rodriguez-Tomé (Hector).- Le Moi et l'Autre dans la conscience de l'adolescent, Paris, Delachaux et Niestlé, 1972.

Rui (Jian R.), Stefanone (Michael A.).- Strategic self-presentation online: a cross-cultural study, Computers in Human Behavior, 29, 2013, p. 110-118.

Skues (Jason L.), Williams (Ben), Wise (Lisa).- The effects of personality traits, self-esteem, loneliness, and narcissism on Facebook use among university students, Computers in Human Behavior, 28, 6, 2012, p. 2414-2419.

Subrahmanyam (Kaveri), Greenfield (Patricia).- La communication en ligne : les adolescents et les médias, dans Octobre (S.), Sirota (R.), L'enfant et ses cultures. approches internationales, Paris, Ministère de la Culture, La documentation française, 2013, p. 123-157.

Suler (John).- The online Dishinibition Effect, CyberPsychology, 7, 3, 2004, p. 321-326.

Tap (Pierre).- Socialisation et construction de l'identité personnelle, dans Malewska-Peyre (H.), Tap (P.), La socialisation de l'enfance à l'adolescence, Paris, Presses universitaires de France, 1991, p. 49-73. 
Tafani (Eric), Falomir (Juan Manuel), Mugny (Gabriel).- Influence sociale et représentations sociales : études expérimentales sur le groupe d'amis idéal, Perspectives cognitives et conduites sociales, 7, 2000, p. 95-124.

Tisseron (Serge).- Intimité et extimité, Communications, 88, 2011, p. 83-91.

Vasquez-Bronfman (Ana), Martinez (Isabel).- La socialisation à l'école. Approche ethnographique, Paris, Presses universitaires de France, 1996.

Walther (Joseph B.).- Selective self-presentation in computer-mediated communication: Hyperpersonal dimensions of technology, language, and cognition, Computers in Human Behavior, 23, 2007, p. 2538-2557.

Wängqvist (Maria), Frisén (Ann).- Who am I Online? Understanding the Meaning of Online contexts for Identity development, Adolescent Research Review, 1, 2, 2016, p. 139-151.

Zimmermann (Grégoire), Quartier (Vincent).- Construction identitaire et relations familiales à l'aune de l'intersubjectivité, dans Moro (C.), Müller Mirza (N.), Roman (P.), L'intersubjectivité en question. Agrégat ou nouveau concept fédérateur pour la psychologie?, Lausanne, Antipodes, 2013, p. 301-321. 


\begin{tabular}{llll}
\hline Variables & & $\boldsymbol{N}$ & $\%$ \\
\hline \multirow{2}{*}{ Âge } & $11-12$ ans & 20 & $42,5 \%$ \\
& $13-15$ ans & 27 & $57,5 \%$ \\
\hline \multirow{2}{*}{ Outils } & Réseaux sociaux & 26 & $55,3 \%$ \\
& Messageries instantanées & 21 & $44,7 \%$ \\
\multirow{2}{*}{ Expression de soi } & Réservée (1 publication/semaine) & 23 & $48,9 \%$ \\
& Expansive (+ de 3 publications/semaine) & 24 & $51,1 \%$ \\
\multirow{2}{*}{ Mode relationnel en ligne } & Restreint (<100 contacts) & 19 & $40,4 \%$ \\
& Large (>100 contacts) & 28 & $59,6 \%$ \\
\hline
\end{tabular}

Tableau 1. Variables illustratives 


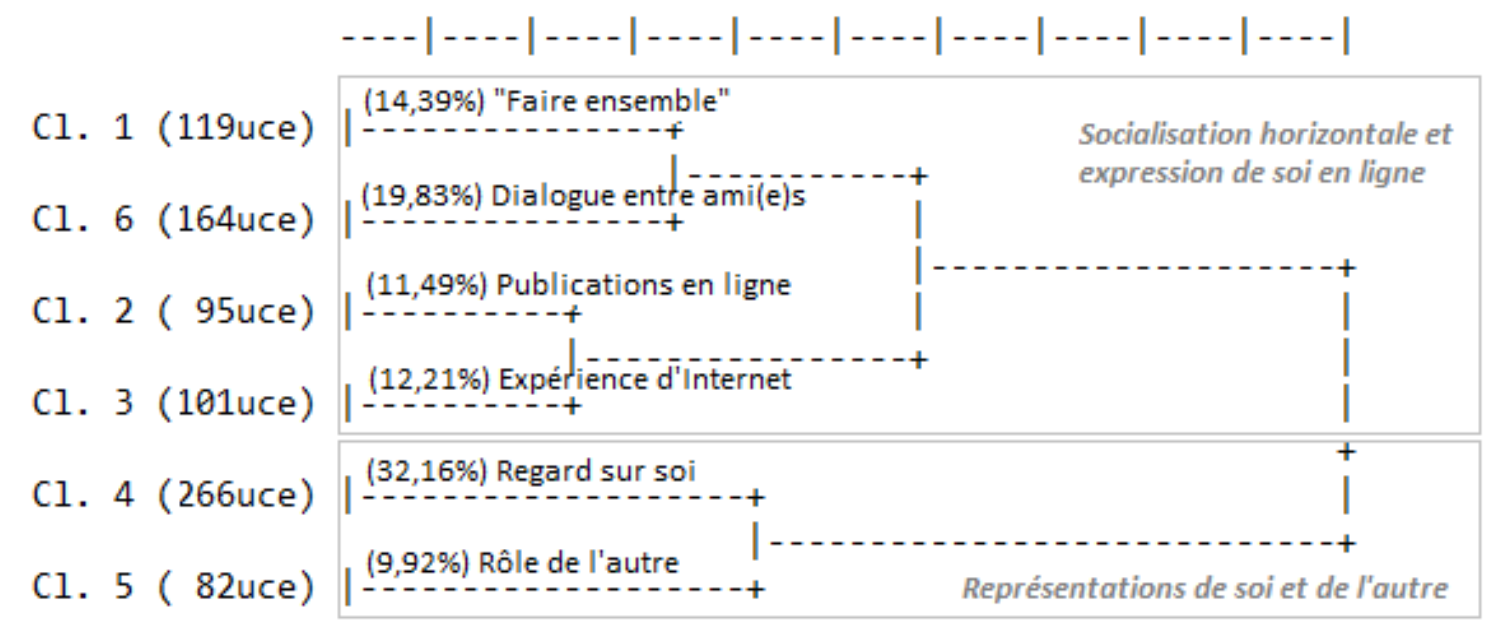

Figure 1. Dendrogramme récapitulatif de l'analyse lexicométrique 